\title{
SUBNANOWATT MICROBUBBLE PRESSURE TRANSDUCER
}

\author{
C. A. Gutierrez ${ }^{*}$ and E. Meng
}

University of Southern California, Los Angeles, California, USA

\begin{abstract}
We present the first all-Parylene microbubble pressure transducer $(\mu \mathrm{BPT})$. The $\mu \mathrm{BPT}$ utilizes an electrolytically generated microbubble $(\mu \mathrm{B})$, trapped in a Parylene microchamber, for pressure sensing. Pressure-induced bubble size variation is detected by electrochemical impedance measurement. Real-time hydrostatic pressure measurement with excellent sensitivity $(-10.7$ $\Omega / \mathrm{psi}, \pm 0.1 \mathrm{psi}$ ) up to $1.8 \mathrm{psi}$ and tracking of internal pump pressure in a MEMS-based implantable drug delivery system were achieved. The transduction method and device format uniquely leverage the surrounding liquid environment, obviating the need for hermetic or special packaging techniques for sensing in wet environments. Arrayed $\mu$ BPTs are biocompatible, fabricated on flexible substrates, ultra-miniature $(200 \mu \mathrm{m}$ diameter, $10 \mu \mathrm{m}$ thick), and can be operated at very low power $(\leq \mathrm{nW})$ making them especially attractive for in vivo pressure measurement applications.
\end{abstract}

\section{INTRODUCTION}

It is known that gas bubbles respond to external pressure [1] but few efforts exploit this phenomena for sensing. At frequencies below resonance $(55 \mathrm{kHz}$ for a bubble of $50 \mu \mathrm{m}$ radius [2]), microbubbles respond instantaneously to pressure variations. Using microelectromechanical systems (MEMS) technology, miniature devices which can precisely produce, localize, and measure bubble response at very small scales can be fabricated. Polymer-based MEMS fabrication technology in combination with microbubble dynamics are leveraged here to introduce a new class of pressure transducer with unique capabilities.

State-of-the-art ultra-miniature pressure sensing technologies generally occupy $400-500 \mu \mathrm{m}$ diameter footprints. Our unique electrochemical-based pressure sensing technique does not utilize conventional diaphragm-based pressure transduction, enabling significant reductions in overall footprint, materials processing costs, and power consumption. Electrochemical impedance-based sensing can be accomplished at nanowatt power levels, making this attractive for wireless and implantable applications. Previously, pressure measurements within a microfluidic channel were demonstrated using $\mu \mathrm{B}$-based transduction [3], however this approach utilized rigid substrate materials and thus was not amenable to wide application (e.g. sensing small pressure variations in vivo). Here, Parylene $\mathrm{C}$ is featured as both the flexible substrate and structural material; this combination enables a novel sensor configuration capable of controlling bubble generation, localization, and transduction in a completely released and portable device compatible with diverse liquid environments. In particular, these features address the unmet need for robust in vivo pressure sensors. Our $\mu \mathrm{B}$ pressure sensing approach eliminates the need for hermetic packaging (for operation in wet environments) and the exclusive use of polymer materials reduces cost, adds mechanical flexibility, and facilities integration for medical applications.

\section{THEORY}

\section{Microbubble Dynamics}

The response of suspended bubbles to changes in ambient pressure is well studied with regard to bubble dynamics and mass diffusion processes across the gas-liquid interface. The fundamental dynamics of a suspended bubble fixed in an unbounded incompressible viscous liquid are governed by the
Rayleigh-Plesset equation [4]:

$$
r \ddot{r}+\frac{3}{2} \dot{r}^{2}=\frac{1}{\rho}\left(p_{g}-p_{\infty}-\frac{2 \sigma}{r}-\frac{4 \mu}{r} \dot{r}\right)
$$

where $r$ is the bubble radius (dots denote time-derivative), $p_{g}$ and $p_{\infty}$ are the pressure in the gas at the bubble wall and ambient pressure far away from the bubble (infinite distance), $\sigma$ is surface tension, $\mu$ is fluid viscosity, and $\rho$ is fluid density.

\section{Bubble generation}
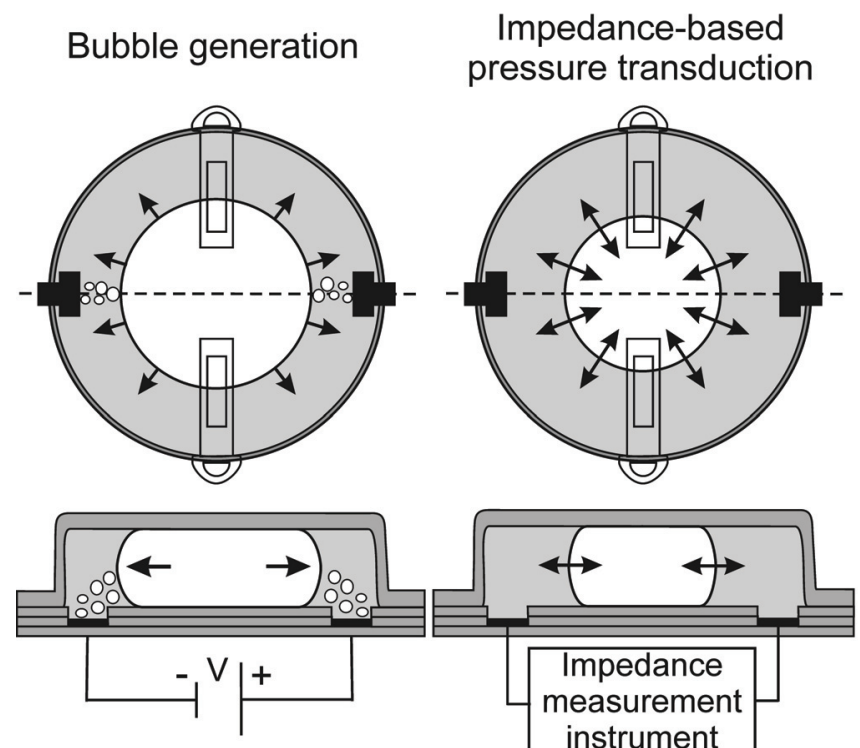

Generated gas $\square$ Parylene-C

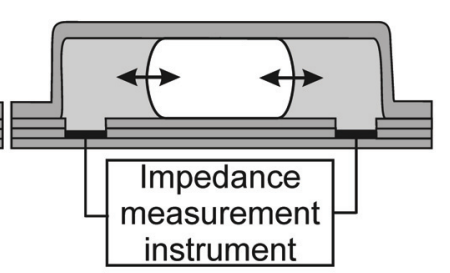
Figure 1: (Left) Electrolytic generation and growth of a bubble within a fluid-filled Parylene microchamber. (Right) Bubble size instantaneously responds to variations in external pressure and is tracked through measurement of solution impedance. Two electrodes are located at the periphery of the chamber and shown in black. Top row = top view, bottom row = cross-sections.

Under the condition that the pressure oscillation frequency is much smaller than the bubble resonant frequency and neglecting any interfacial mass transfer effects, a simple assumption is that the gas follows a polytropic law of compression with a polytropic exponent $b \approx 1$ at low frequency [4]:

$$
p_{g}=p_{i}\left(\frac{r_{0}}{r}\right)^{b}
$$

where $r_{0}$ and $p_{i}$ are initial bubble radius and equilibrium gas pressure inside the bubble, respectively. From Eq. 1, the following pressure-radius relationship is obtained:

$$
p_{\infty}=p_{i}\left(\frac{r_{0}}{r}\right)^{b}-\frac{2 \sigma}{r}
$$

The pressure-radius bubble response can therefore be estimated by Eq. 3. which describes the balance of forces acting on the bubble. For a given number of gas molecules produced by 
electrolysis and a non-varying surface tension, bubble size is a direct and instantaneous measure of ambient pressure (Figure 1).

If one now considers the bubble behavior over larger time scales, the effects of mass transfer at the bubble liquid interface become significant. The bubble will dissolve in the presence of an under-saturated liquid as a consequence of diffusion across the bubble-liquid interface. Bubble dissolution rate in an undersaturated solution was derived by Epstein and Plesset [1]:

$$
\frac{d r}{d t}=\frac{k\left(c_{s}-c_{\infty}\right)}{\rho_{\infty}+2 \tau / 3 r}\left(\frac{1}{r}+\frac{1}{(\pi k t)^{1 / 2}}\right)
$$

where $k$ is the coefficient of diffusivity of the gas in the liquid, $c_{s}$ and $c_{\infty}$ are the saturation concentrations of gas in the liquid at the bubble surface and in the bulk, $\rho_{\infty}$ the density of gas in the bubble with a gas-liquid interface of zero curvature, and $\tau$ is the modified surface tension. The mass-diffusion processes taking place across the bubble-liquid interface play a significant role in the behavior of gas bubbles because they may ultimately determine the presence or absence of bubbles in a liquid. These processes are governed by Henry's law, which establishes a connection between the partial pressure of a gas acting on a liquid surface, $p_{g}$, and the equilibrium (or saturation) concentration of gas in the liquid:

$$
c_{s}=a p_{g}
$$

$a$ is a constant characteristic of the particular gas-liquid combination and is a function of temperature. If the ambient pressure is fixed and equal to $p_{\infty}$, then it is clear that unless the gas concentration $c$ at the bubble surface satisfies Eq. 5, the bubble will not be in equilibrium, and will either grow or shrink according to whether $c>c_{s}$ or $c<c_{s}$. Mass transfer driven bubble dissolution is a slow process; typical values for bubbles of radius $\sim 100 \mu \mathrm{m}$ are estimated to be on the order of $\sim 0.1 \mu \mathrm{m} / \mathrm{sec}$ [4].

\section{Liquid Impedance-based Transduction}

The underlying principle of impedance-based transduction is the measurement of solution resistance. The electrode-electrolyte interface has been well studied and characterized $[5,6]$ and is simply modeled as shown in Figure 2 below:

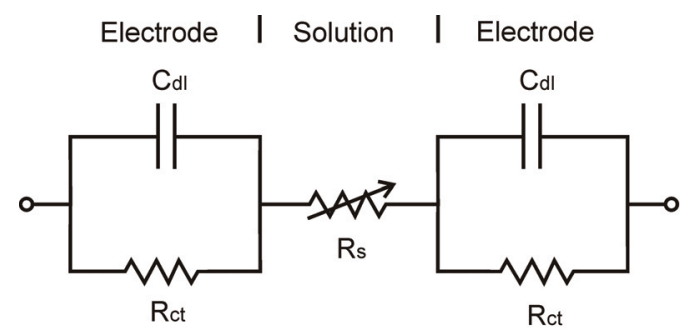

Figure 2: Electrical equivalent circuit for the electrode-electrolyte interface (Randles model).

where $C_{d l}, R_{c t}$, and $R_{s}$ are the double-layer capacitance, charge transfer resistance, and solution resistance, respectively. By applying a small alternating current at a sufficiently high frequency (typically $>1 \mathrm{kHz}$ ), the parallel components are shorted through $C_{d l}$, and $R_{s}$ dominates the measured impedance. The solution resistance is a function of ionic concentration, distance between the electrodes, and cross-sectional area of electrolyte between the electrodes. A trapped $\mu \mathrm{B}$ acts as an insulating sphere in the conductive incompressible electrolyte between the electrodes. Therefore, monitoring $\mu \mathrm{B}$ size variations, through measurement of electrolyte impedance (resistance), is a direct measure of external pressure variations.

\section{METHODS}

\section{Materials and Fabrication}

The transducer features an all-Parylene construction (substrate and structural material) which provides flexibility and biocompatibility for medical and in vivo applications. Electrodes and traces are formed from thin film platinum. No further materials were utilized nor were any hermetic sealing or encapsulation techniques required. This "wet" sensor is open to and filled by the surrounding aqueous liquid (via fluidic access ports) which serves as the electrolyte (Figure 3). Isolation of the sensor from the environment is therefore not necessary. Added costs and complexities associated with hermetic packaging are avoided.
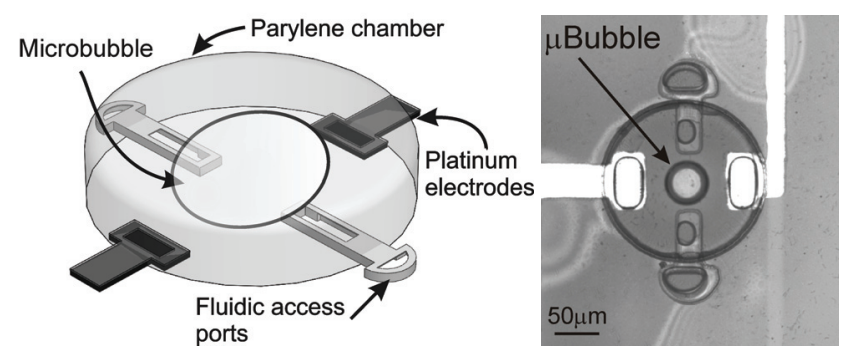

Figure 3: (Left) Model of pressure transducer. (Right) Optical micrograph of actual device (200 $\mathrm{um}$ diameter) with a bubble.

Fabrication techniques were previously reported [7]. Briefly, platinum electrodes $(2000 \AA)$ were deposited and patterned on a Parylene coated $(10 \mu \mathrm{m}$ thick) soda lime wafer (1 mm thick) followed by a $1 \mu \mathrm{m}$ thick Parylene insulation layer. A $2 \mu \mathrm{m}$ sacrificial photoresist layer then formed the fluidic access ports followed by a second Parylene deposition step ( $2 \mu \mathrm{m}$ thick). A $10-$ $12 \mu \mathrm{m}$ sacrificial photoresist layer was then spun-on and patterned to establish the chamber height and diameter $(200-300 \mu \mathrm{m})$. The chamber structure was enclosed by a final of $4.2 \mu \mathrm{m}$ Parylene layer, followed by opening of access port vias by oxygen plasma.

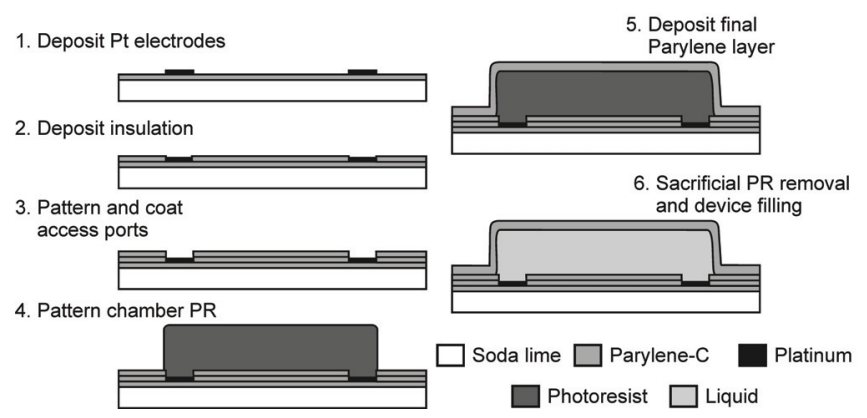

Figure 4: Fabrication process flow for a single transducer. Crosssection taken through electrodes.

Following removal of sacrificial photoresist by immersion in acetone and isopropyl alcohol, chambers were simultaneously filled at the wafer-level by passive soaking in a bath of the desired electrolyte solution. The chamber design allows the ionic conductive path to be constrained within in the chamber interior. This enables the measurement solution resistance in a known geometry and minimizes the potential for cross talk between arrayed sensors. Test dies containing several $\mu$ BPTs were 
fabricated. In addition, a $\mu \mathrm{BPT}$ array integrated at the tip of flexible Parylene cable was fabricated and completely released from the substrate to form a pressure probe.

\section{Experimental Setup}

The devices were filled with deionized (DI) water or $1 \mathrm{x}$ phosphate buffered saline (PBS) as the electrolyte. A microbubble was electrolytically generated within the chamber by applying a DC current pulse (typically $1 \mu \mathrm{A}, 5 \mathrm{~s}$ for DI water). Device impedance was monitored using a LabVIEW-interfaced Analog Devices Impedance Converter (AD5933) calibrated using an Agilent Precision LCR meter $(1 \mathrm{~V}$ pp, $5 \mathrm{kHz})$. A calibrated pressure source metered external hydrostatic pressure applied to $\mu$ BPTs housed in a testing jig. A series of pressure steps were applied up to 1.8 psi while impedance was monitored.

\section{RESULTS AND DISCUSSION}

\section{Hydrostatic Pressure Measurement}

Measurement of hydrostatic pressure variations was performed. Impedance measurements exhibited excellent resolution up to 1.8 psi (Figure 5).

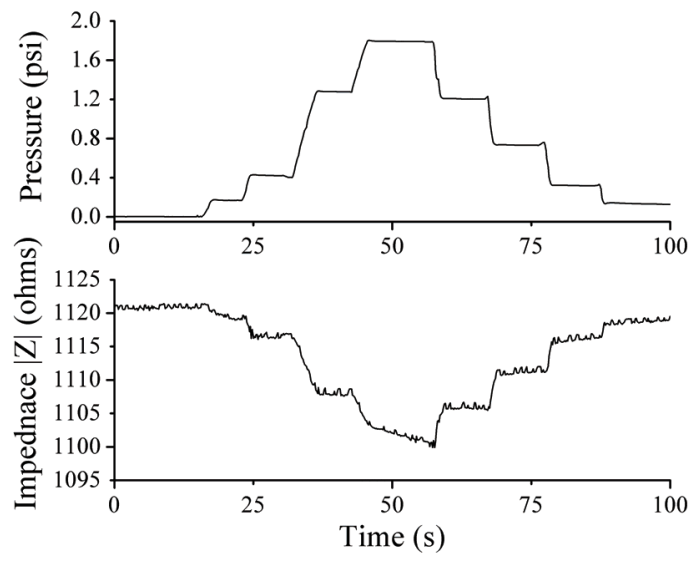

Figure 5: (Top) Profile of applied hydrostatic pressure from calibrated pressure source. (Bottom) The measured impedance response (1x PBS) from the $\mu B P T$ to the applied pressure profile.

Measurements were obtained within 100 seconds of bubble formation; minimal change in bubble volume occurred over this time. Some measurement drift was observed at maximum pressure (1.8 psi) and was attributed to mass transport-driven bubble dissolution at this elevated pressure. Stable measurements were most easily obtained at pressures below 2 psi $(13.9 \mathrm{kPa})$. A calibrated sensor response of $-10.7 \Omega /$ psi was obtained by a best fit linearization and clearly demonstrates pressure tracking capability (Figure 6). Pressure measurement resolution was estimated to be $\pm 0.1 \mathrm{psi}(689.4 \mathrm{~Pa})$. It was possible to detect pressure variations with excitation amplitudes of only $100 \mathrm{mV}, 10 \mathrm{nA}(\sim 1 \mathrm{nW})$; detection at lower excitation amplitudes was also possible but not characterized here. When DI water was used as the electrolyte, a solution resistance of several megaohms was typically observed making it possible to realize sub-nanowatt device operation. The bubble generation phase does require somewhat higher power levels but these are typically in the microwatt range and only a few seconds in duration. Although a device configuration open the surrounding environment is desirable the integration of liquid entrapment structures, such as a Parylene-based stiction valve, can provide a means for the entrapment of a particular electrolyte within the device while it is operated in a different medium [8]. This capability, combined with the small size and polymer construction, makes this technology amenable to low power applications such as implantable wireless pressure monitoring.

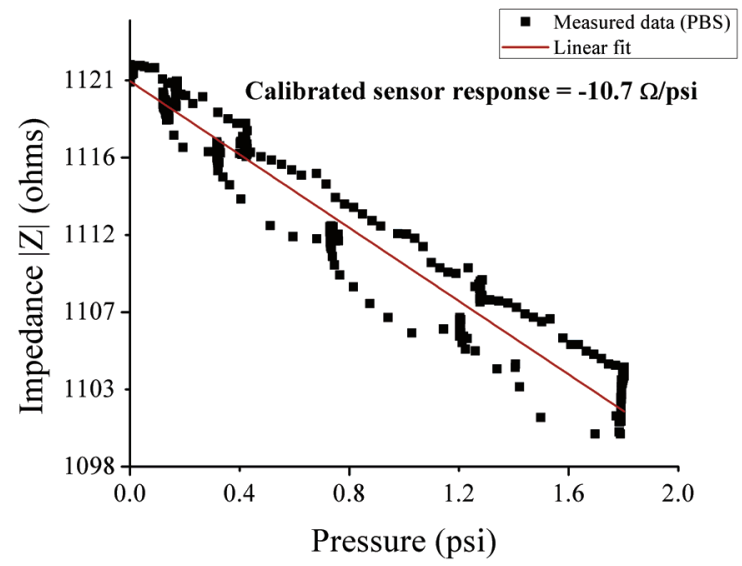

Figure 6: Impedance-pressure plot generated from data in Fig. 5

\section{Drug Delivery Pump Pressure Measurement}

For practical applications, an array of $\mu$ BPTs was fabricated on the tip of a flexible Parylene cable. This allowed placement of transducers in an environment of choice (Figure 7).

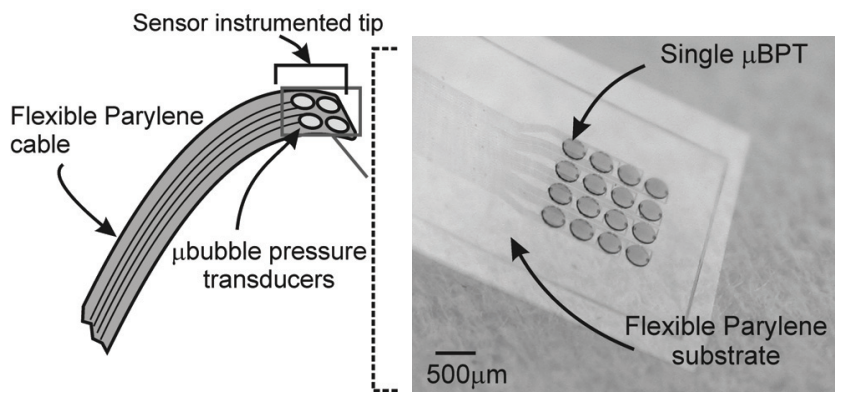

Figure 7: (Left) Drawing of $\mu B P T$ array positioned at the tip of a flexible Parylene cable. (Right) Optical micrograph of array.

Practical pressure sensor operation was demonstrated in the real-time tracking of internal pressure in an electrolysis pump utilized in a MEMS drug delivery system [9]. Unlike conventional transducers, the small form factor and flexibility of the $\mu \mathrm{BPT}$ array made possible its integration within the polymer-based drug delivery pump to assess internal pressurization conditions. The $\mu$ BPT array was placed in contact with fluid in the pump chamber. (Figure 8). Pump ON/OFF states were clearly detected and calibrated pressure measurements agree well with previously reported data measured with a conventional silicon pressure transducer [9] (Figure 9).

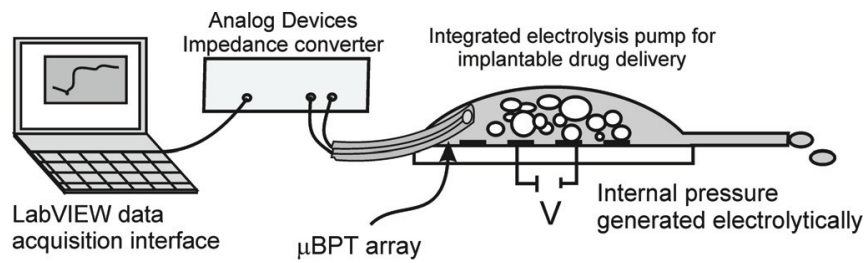

Figure 8: Experimental setup for monitoring intra-pump pressure produced via electrolysis. 


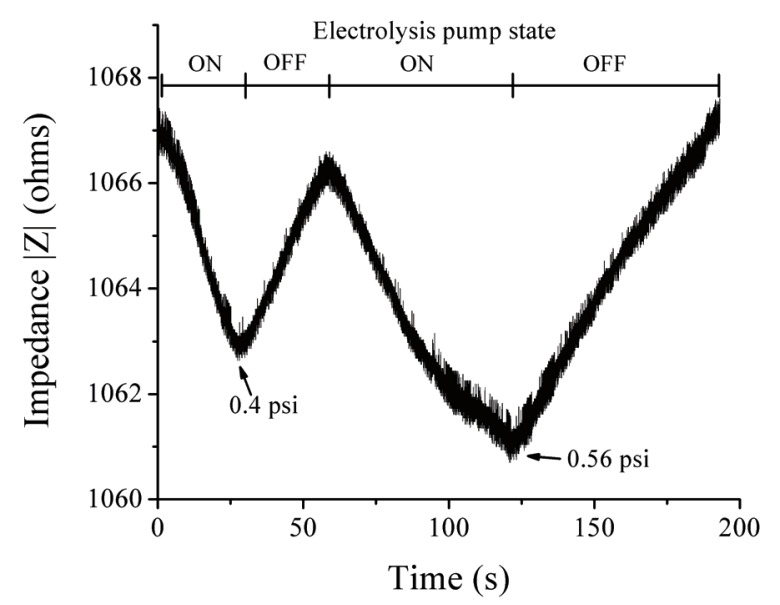

Figure 9: Impedance response to electrolysis pump activation/deactivation cycles (5mA pump current). Calibrated measurement of pressure (-10.7 $\Omega /$ psi) are shown at two points.

\section{Static Bubble Approximation}

According to Faraday's law, the number of gas molecules in an electrolytic gas bubble is determined by the total charge transferred during electrolysis:

$$
\Delta Q=n z F
$$

where $z$ is the total charge associated with the formation of a single gas molecule, $F$ is the Faraday constant, and $n$ is moles of gas molecules. The electrochemical reaction of electrolytic bubble generation was thoroughly studied in [10]. It was shown that $\mathrm{H}_{2}$ and $\mathrm{Cl}_{2}$ gases are the main products of electrolysis of a $\mathrm{NaCl}$ aqueous solution with negligible byproducts. In practice, the gas composition of the bubble is nearly all hydrogen, due to the lower standard potential required for hydrogen gas evolution. Additionally, the solubility of hydrogen is about three orders of magnitude lower than for $\mathrm{Cl}_{2}$.

Once the bubble is formed, the slow diffusion-limited dissolution process maintains an approximately constant bubble size for a period of time ( $20 \mathrm{~min})$, and is dependent on the kinetics and solubility of the gas/liquid interface. There are several factors enabling the use of a slowly shrinking bubble to measure pressure. Generally, smaller bubbles will have higher surface tension and therefore higher internal pressures which serves to speed up the dissolution according to Eq 1. The surface to volume ratio also increases with decreasing bubble size. Finally, it is known that platinum acts as a catalyst and can increase the recombination rate significantly.

In this device, the exposure of the platinum electrodes is limited to the periphery and there is essentially no contact between the recombining gas and the electrodes, thereby minimizing any catalytic effect. In addition, the bubble is compressed due to the fixed chamber height and is forced to expand only in lateral directions. This effectively reduced the surface to volume ratio over which bubble dissolution can occur by limiting mass transport processes to the edges of the bubble. In other words, the top and bottom surfaces of the bubble, which are in contact with the chambers top and bottom surfaces, do not contribute significantly to the mass transport process. These factors taken together give rise to longer bubble lifetimes allowing their use as quasi-constant pressure sensors over short to medium time scales ( 15-20 min). Over larger time scales, the measurement of pressure via bubble dissolution rate is also possible and was previously demonstrated
[3]. Other electrolyte-gas combinations can be utilized, such as ethylene glycol or alcohols, which may provide insoluble conditions for entrapped gases for applications requiring long term constant-volume $\mu \mathrm{B}$ transduction capability.

\section{CONCLUSION}

We have designed and fabricated a Parylene-based pressure transducer utilizing a unique liquid-impedance transduction technique. Response of a bubble to external pressure variations is harnessed to electrochemically monitor pressure. The sensor operates in communication with the surrounding liquid environment (either DI water or PBS), thereby eliminating the need for hermetic packaging. Preliminary sensor characterization indicates a sensing resolution of \pm 0.1 psi over a 0 - 2 psi range.

\section{ACKNOWLEDGEMENTS}

This work was funded in part by the Engineering Research Centers Program of the NSF under Award Number EEC-0310723, an NSF CAREER Award (ECS-0547544), and the Bill and Melinda Gates Foundation (CG). The authors would like to thank Dr. Donghai Zhu, Mr. Connor McCarty, Ms. Roya Sheybani and the members of the USC Biomedical Microsystems Laboratory for their assistance.

\section{REFERENCES}

[1] P. S. Epstein and M. S. Plesset, "On the stability of gas bubbles in liquid-gas solutions," J. Chem. Phys., vol. 18, pp. 1505-1509, 1950.

[2] B. Ran and J. Katz, "The response of microscopic bubbles to sudden changes in ambient pressure," J. Fluid Mech., vol. 224, pp. 91-115, 1991.

[3] D. A. Ateya, A. A. Shah, et al., "Impedance-based response of an electrolytic gas bubble to pressure in microfluidic channels," Sensors and Actuators A, vol. 122, pp. 235-241, 2005.

[4] M. S. Plesset and A. Prosperetti, "Bubble dynamics and cavitation," Ann. Rev. Fluid Mech., vol. 9, pp. 145-185, 1977.

[5] J. E. B. Randles, "Kinetics of rapid electrode reactions," in Proc. Discussions of the Faraday Society 1947, pp. 11-19.

[6] H. L. Helmholtz, "Studien uber electricshe grenzschichten," Annalen der Physic und Chemie, vol. 7, 1879.

[7] C. A. Gutierrez, C. McCarty, et al., "An Implantable AllParylene Liquid-Impedance based MEMS Force Sensor," in Proc. IEEE MEMS 2010, Hong Kong, China, pp. 600-603.

[8] C. A. Gutierrez and E. Meng, "Improved Self-Sealing Liquid Encapsulation in Parylene Structures by Integrated Stackable Annular-Plate Stiction Valve," in Proc. IEEE MEMS 2010, Hong Kong, China, pp. 524-527.

[9] P.-Y. Li, R. Sheybani, et al., "A Parylene Bellows Electrochemical Actuator," Microelectromechanical Systems, Journal of, vol. 19, pp. 215-228, 2010.

[10] S. Z. Hua, F. Sachs, et al., "Microfluidic actuation using electrochemically generated bubbles," Anal. Chem., vol. 74, pp. 6392-6396, 2002.

\section{CONTACT}

*C. A. Gutierrez., tel: (213) 821 3949; cagutier@usc.edu 